\title{
Perfil epidemiológico, clínico e laboratorial do exame citopatológico realizado em Espinosa, Minas Gerais, durante o ano de 2014
}

\author{
Patrick Leonardo Nogueira da Silva \\ Professor da Escola Técnica de Saúde da Universidade Estadual de Montes Claros - UNIMONTES \\ Enfermeiro especialista em Saúde da Família e Enfermagem do Trabalho \\ Mestrando em Saúde, Sociedade e Ambiente pela Universidade Federal dos Vales do Jequitinhonha e Mucuri - \\ UFVJM \\ $\triangle$ patrick_mocesp70@hotmail.com
}

Recebido em 24 de fevereiro de 2018

Aceito em 9 de agosto de 2018

\section{Resumo:}

Objetivou-se investigar o perfil epidemiológico, clínico e laboratorial do exame citopatológico cérvico-vaginal realizado no município de Espinosa. Trata-se de um estudo descritivo, exploratório, retrospectivo, documental, com abordagem quantitativa, sendo a amostra composta por dados do Departamento de Informática do Sistema Único de Saúde (DATASUS) referente a 135 coletas citopatológicas realizadas no ano de 2014. O tratamento dos dados se deu por meio de análise estatística univariada. Observou-se que 72,5\% foram coletadas em um intervalo de 0-10 dias. Em 77\%, o intervalo para a conclusão dos resultados foi estabelecido entre 11-20 dias. 0 tempo do exame foi de 0-30 dias (75,5\%). Quanto à cor/raça, todos os registros não apresentavam esta informação. 0 intervalo etário mais prevalente foi de 40-49 anos (32,4\%). O tempo do último exame preventivo foi de um ano (49,6\%). Todas as amostras encontravam-se satisfatórias à análise. Em 58,5\%, os resultados entrados estavam dentro da normalidade. Portanto, os resultados das amostras coletadas atendem satisfatoriamente ao perfil de prevenção do câncer de colo uterino nas mulheres, porém há falhas no preenchimento dos dados na ficha de coleta por parte do profissional.

Palavras-chave: Neoplasias do colo do útero, Prevenção secundária, Testes diagnósticos de rotina, Teste de Papanicolaou.

\section{Epidemiological profile, clinical and laboratory of cytopathological examination performed in Espinosa, Minas Gerais, during the year of 2014}

\begin{abstract}
:
The objective of this study was to investigate the epidemiological, clinical and laboratory profile of cervical-vaginal cytopathology performed in the city of Espinosa. This is a descriptive, exploratory, retrospective, documental study, with a quantitative approach, being the sample composed by data from the Department of Informatics of the Unified Health System (DATASUS) referring to 135 cytopathological collections performed in 2014. The treatment of this was done through univariate statistical analysis. It was observed that $72.5 \%$ were collected in a range of $0-10$ days. In $77 \%$, the interval for the conclusion of the results was established between 11-20 days. The time of the examination was 0-30 days (75.5\%). Regarding color/race, all records did not present this information. The most prevalent age range was $40-49$ years $(32.4 \%)$. The time of the last preventive examination was one year (49.6\%). All samples were satisfactory for analysis. In 58.5\%, the results entered were within the normal range. Therefore, the results of the samples collected satisfactorily meet the profile of cervical cancer prevention in women, but there are failures in filling the data in the collection form by the professional.

Keywords: Uterine cervical neoplasms, Secondary Prevention, Diagnostic tests, routine, Papanicolaou test.
\end{abstract}




\section{Perfil epidemiológico, clínico y de laboratorio de citología realizado en Espinosa, Minas Gerais, durante el año de 2014}

\section{Resumen:}

Se objetivó investigar el perfil epidemiológico, clínico y de laboratorio del examen citopatológico cérvico-vaginal realizado en el municipio de Espinosa. Se trata de un estudio descriptivo, exploratorio, retrospectivo, documental, con abordaje cuantitativo, siendo la muestra compuesta por datos del Departamento de Informática del Sistema Único de Salud (DATASUS) referente a 135 colectas citopatológicas realizadas en el año 2014. El tratamiento los datos se dieron por medio de análisis estadístico univariado. Se observó que 72,5\% fueron recolectadas en un intervalo de 0-10 días. En un 77\%, el intervalo para la conclusión de los resultados se estableció entre 11-20 días. El tiempo del examen fue de 0-30 días (75,5\%). En cuanto al color/raza, todos los registros no presentaban esta información. El intervalo de edad más prevalente fue de 40-49 años (32,4\%). El tiempo del último examen preventivo fue de un año (49,6\%). Todas las muestras se encontraban satisfactorias al análisis. En el 58,5\%, los resultados entrados estaban dentro de la normalidad. Por lo tanto, los resultados de las muestras recolectadas atienden satisfactoriamente al perfil de prevención del cáncer de cuello uterino en las mujeres, pero hay fallas en el llenado de los datos en la ficha de recolección por parte del profesional.

Palabras clave: Neoplasias del cuello uterino, Prevención secundaria, Pruebas diagnósticas de rutina, Prueba de Papanicolaou.

\section{INTRODUÇÃO}

O exame de Prevenção de Câncer Cérvico Uterino (PCCU), também conhecido como esfregaço cervicovaginal, colpocitologia oncótica cervical ou "Papanicolau", é um teste de detecção de alterações em células cervicais uterinas. Configura-se a principal estratégia de diagnóstico precoce de lesões intra-epiteliais (LIE), antes que a mulher apresente sintomas. Realiza-se em instituições de Atenção Primária à Saúde (APS) com profissionais capacitados, sendo estes o médico e o enfermeiro, de modo que os mesmos orientem a mulher sobre as diretrizes do exame, bem como a sua importância, pois a periodicidade de sua realização permite a antecipação do diagnóstico e a redução da mortalidade por câncer do colo do útero (CCU). O PCCU é indolor, simples e rápido (BRASIL, 2015a).

Em um estudo científico o qual objetivou discutir o fluxo assistencial de mulheres com resultado do exame citopatológico alterado em uma rede de atenção à saúde, foi observado inconformidade entre a conduta/intervenção profissional mediante os resultados dos exames obtidos. Em 78\% das lesões não neoplásicas de baixo grau houve o encaminhamento da paciente para tratamento com ginecologista em instituição de alta complexidade, enquanto que $21 \%$ das lesões neoplásicas de alto grau que deveriam ser encaminhadas para o tratamento em instituição de alta complexidade são tratadas no nível primário. Há falta de comunicação entre os pontos de atenção, sendo estes pontos de atenção primária (baixa 
complexidade), secundária (média complexidade) e terciária (alta complexidade), e ausência de protocolos de referência e contra-referência. Em 18 (54,5\%) unidades de atenção básica, as mulheres buscam espontaneamente o serviço de regulação (FARIAS; BARBIERI, 2016).

Em um inquérito realizado na cidade de Juiz de Fora, Minas Gerais (MG), no ano de 2010, a prevalência de não submissão da mulher ao exame foi de 21,3\% em decorrência da não realização da busca ativa, bem como pela dificuldade geográfica tendo em vista que muitas destas mulheres residem em áreas distantes com pouca acessibilidade ao serviço de saúde. Entre as mulheres com 25 anos ou mais, a prevalência foi de 15,1\%. A não adesão foi mais frequente entre as mulheres jovens, solteiras e com baixa escolaridade. Esta, por sua vez, se manteve associada ao desfecho, indicando que mulheres com mais anos de estudo têm maiores chances de realizarem o exame. O contato com o serviço de saúde para realização do pré-natal não foi determinante para garantir o acesso ao exame, indicando falha do rastreamento em regiões onde o mesmo é fundamental (RIBEIRO et al., 2016).

Este estudo objetivou investigar o perfil epidemiológico, clínico e laboratorial do exame citopatológico cérvico-vaginal e da microflora realizado durante o ano de 2014 no município de Espinosa, Minas Gerais.

\section{MÉTODO}

Trata-se de um estudo descritivo, exploratório, retrospectivo, documental, com abordagem quantitativa, realizado no município de Espinosa, localizado no norte do Estado de Minas Gerais. A amostra deste estudo foi composta por 135 coletas de exames citopatológicos cérvico-vaginal realizados por mulheres do referido município durante o período de janeiro a dezembro de 2014.

A cidade de Espinosa encontra-se localizada no extremo norte do Estado de Minas Gerais. Apresenta uma população de 31.113 habitantes, área de 1.868,970 km² e um bioma regido pelo cerrado, caatinga e mata atlântica. Apresenta total cobertura de Estratégia de Saúde da Família (ESF) na qual atendem a sua população (INSTITUTO BRASILEIRO DE GEOGRAFIA E ESTATÍSTICA [IBGE], 2016). O município dispõe de um contingente populacional 
feminino, com idade entre 15 anos e maiores de 60 anos, de 12.622 mulheres, equivalendo a $40,5 \%$ da população total, que se encontra em idade fértil e apta a realizar o exame preventivo de câncer de colo uterino (PCCU) (BRASIL, 2015b).

Foram adotados os seguintes critérios de inclusão para participação no estudo: amostras coletadas de mulheres que tenham realizado o exame preventivo durante o ano de 2014, sendo este período escolhido por conveniência, tendo este o maior quantitativo de realização de exames com relação aos anos anteriores. Neste ano houve o aumento da intensificação das ações de promoção e prevenção com relação ao câncer de colo uterino, bem como a intensificação da busca ativa das mulheres faltantes as quais apresentavam mais de um ano quanto à realização do último exame citopatológico.

Foi utilizado um formulário estruturado de elaboração própria baseado na ficha de coleta do exame como instrumento de coleta de dados. 0 mesmo apresentava as seguintes variáveis de coleta: intervalo da coleta, intervalo do resultado, tempo do exame, cor/raça, faixa etária, tempo do último preventivo e adequabilidade do material.

A coleta dos dados foi realizada por meio do Departamento de Informática do Sistema Único de Saúde (DATASUS) na qual os dados são disponibilizados publicamente via online (http://tabnet.datasus.gov.br/cgi/deftohtm.exe?siscolo/ver4/DEF/uf/MGCCOLO4.def). Os dados foram apresentados em tabelas com frequências absolutas e percentuais elaboradas por meio do Microsoft Excel ${ }^{\circledR}$, versão 2010. O tratamento dos dados foi feito por meio de análise estatística uni-variada. Esta inclui todos os métodos de Estatística Descritiva que permitem a análise de cada variável separadamente com Intervalo de Confiança de 95\% ( $p \geq$ 0,05). Foi aplicado o Epi Info, programa estatístico de acesso público.

Por se tratar de dados de domínio público, não foi necessário/obrigatório o envio do mesmo para análise e aprovação do Comitê de Ética em Pesquisa (CEP).

\section{RESULTADOS}

O perfil dos exames citopatológicos pode ser observado conforme os resultados da Tabela 1. Observa-se eficiência no tempo de coleta das amostras, bem como no tempo de 
liberação dos resultados, tendo em vista a adequabilidade do material na coleta tornando-as satisfatórias à análise. Destas, detectou-se lesão intra-epitelial (LIE) de alto grau e carcinoma epidermóide invasor (CEI) em 1\% das amostras.

Observou-se que, em $58,5 \%$ das amostras, os resultados encontrados estavam dentro da normalidade da microflora vaginal, apresentando como microrganismo dominante o Lactobacillus sp. Houve também a prevalência de: bacilos supracitoplasmáticos (gardinerella) $(25,1 \%)$ e cocos $(24,4 \%)$ (Tabela 2$)$. 
Patrick Leonardo Nogueira da Silva

Tabela 1 - Perfil clínico-epidemiológico dos exames citopatológicos realizados em 2014 no município de Espinosa. Espinosa, MG, 2017. (n=135)

\begin{tabular}{|c|c|c|c|c|c|c|c|c|}
\hline \multirow{2}{*}{ Variáveis } & \multicolumn{2}{|c|}{ Quantidade de exames } & \multicolumn{2}{|c|}{ LIE-BG } & \multicolumn{2}{|c|}{ LIE-AG } & \multicolumn{2}{|c|}{ CEI } \\
\hline & $\mathbf{n}$ & $\%$ & $\mathbf{n}$ & $\%$ & $\mathbf{n}$ & $\%$ & $\mathbf{n}$ & $\%$ \\
\hline \multicolumn{9}{|l|}{ Intervalo da coleta (dias) } \\
\hline $0-10$ & 98 & 72,5 & 00 & 00 & 01 & 1,0 & 01 & 1,0 \\
\hline $11-20$ & 23 & 17,0 & 00 & 00 & 00 & 00 & 00 & 00 \\
\hline $21-30$ & 08 & 5,9 & 00 & 00 & 00 & 00 & 00 & 00 \\
\hline$>30$ & 06 & 4,6 & 00 & 00 & 00 & 00 & 00 & 00 \\
\hline \multicolumn{9}{|l|}{ Intervalo do resultado (dias) } \\
\hline $0-10$ & 29 & 21,4 & 00 & 00 & 00 & 00 & 00 & 00 \\
\hline $11-20$ & 104 & 77,0 & 00 & 00 & 01 & 0,99 & 01 & 0,99 \\
\hline $21-30$ & 02 & 1,6 & 00 & 00 & 00 & 00 & 00 & 00 \\
\hline \multicolumn{9}{|l|}{ Tempo do exame (dias) } \\
\hline $0-30$ & 102 & 75,5 & 00 & 00 & 01 & 0,98 & 01 & 0,98 \\
\hline $31-60$ & 30 & 22,2 & 00 & 00 & 00 & 00 & 00 & 00 \\
\hline$>60$ & 03 & 2,3 & 00 & 00 & 00 & 00 & 00 & 00 \\
\hline \multicolumn{9}{|l|}{ Cor/raça } \\
\hline Sem informação & 135 & 100,0 & 00 & 00 & 01 & 0,74 & 01 & 0,74 \\
\hline \multicolumn{9}{|l|}{ Faixa etária (anos) } \\
\hline $15-19$ & 05 & 3,7 & 00 & 00 & 00 & 00 & 00 & 00 \\
\hline $20-29$ & 16 & 11,7 & 00 & 00 & 00 & 00 & 00 & 00 \\
\hline 30-39 & 27 & 19,9 & 00 & 00 & 00 & 00 & 00 & 00 \\
\hline $40-49$ & 44 & 32,4 & 00 & 00 & 01 & 4,5 & 00 & 00 \\
\hline $50-59$ & 34 & 25,1 & 00 & 00 & 00 & 00 & 01 & 7,1 \\
\hline$>60$ & 09 & 7,2 & 00 & 00 & 00 & 00 & 00 & 00 \\
\hline \multicolumn{9}{|c|}{ Tempo do último preventivo (ano) } \\
\hline Ignorado/Branco & 05 & 3,7 & 00 & 00 & 00 & 00 & 00 & 00 \\
\hline$<1$ & 08 & 5,9 & 00 & 00 & 00 & 00 & 00 & 00 \\
\hline $1-2$ & 92 & 68,1 & 00 & 00 & 01 & 4,0 & 00 & 00 \\
\hline $3-4$ & 12 & 8,8 & 00 & 00 & 00 & 00 & 00 & 00 \\
\hline$\geq 5$ & 07 & 5,1 & 00 & 00 & 00 & 00 & 00 & 00 \\
\hline Não se lembra & 11 & 8,4 & 00 & 00 & 00 & 00 & 00 & 00 \\
\hline \multicolumn{9}{|l|}{ Adequabilidade do material } \\
\hline Satisfatório & 135 & 100,0 & 00 & 00 & 01 & 0,74 & 01 & 0,74 \\
\hline
\end{tabular}

Fonte: TABNET/DATASUS. LIE-BG = Lesão intra-epitelial de baixo grau, LIE-AG = Lesão intra-epitelial de alto grau, CEI = Carcinoma epidermóide invasor. 
Perfil epidemiológico, clínico e laboratorial do exame citopatológico realizado em Espinosa, Minas Gerais, durante o ano de 2014

Tabela 2 - Perfil laboratorial dos resultados dos exames citopatológicos realizados em 2014 no município de Espinosa. Espinosa, MG, 2017. (n=135)

\begin{tabular}{|c|c|c|c|c|c|c|c|c|c|c|}
\hline \multirow{2}{*}{ Município } & \multicolumn{2}{|c|}{ Lactobacillus sp } & \multicolumn{2}{|c|}{ Cocos } & \multicolumn{2}{|c|}{ Trichomonas } & \multicolumn{2}{|c|}{ Gardnerella } & \multicolumn{2}{|c|}{ Outros bacilos } \\
\hline & $\mathbf{n}$ & $\%$ & $\mathbf{n}$ & $\%$ & $\mathbf{n}$ & $\%$ & $\mathbf{n}$ & $\%$ & $\mathbf{n}$ & $\%$ \\
\hline ESPINOSA & 79 & 58,5 & 33 & 24,4 & 03 & 2,2 & 34 & 25,1 & 01 & 0,74 \\
\hline
\end{tabular}

Fonte: TABNET/DATASUS. 


\section{DISCUSSÃO}

O intervalo de tempo repercute na agilidade do resultado do exame. Antes da sua realização, a mulher deve fazê-lo quando não estiver menstruando. O melhor período é entre o $10^{\circ}$ e $20^{\circ}$ dia após o primeiro dia do seu último período menstrual. Por dois dias antes do exame, a mulher deve evitar piscina e banheiras, duchas vaginais, tampões, desodorantes ou medicamentos vaginais, espermicidas e cremes vaginais (a menos que seja recomendado explicitamente pelo seu médico). Estes produtos e situações podem retirar ou esconder as células anormais. A mulher deve também evitar relações sexuais por dois dias antes do exame. Após o exame, a mulher pode voltar a suas atividades normais imediatamente (BAIRROS et al., 2011; DISCACCIATI; BARBOZA; ZEFERINO, 2014).

Um estudo realizado em São Leopoldo e Pelotas, Rio Grande do Sul (RS), objetivou investigar o acesso de mulheres negras e brancas aos exames de detecção precoce de câncer de mama e colo de útero (citopatológico) na qual foram entrevistadas 2.030 mulheres, sendo que $16,1 \%$ eram negras e $83,9 \%$ brancas. A probabilidade de mulheres não realizarem os exames citopatológico e de mama foi significantemente maior nas negras. A desigualdade racial no acesso aos exames de detecção precoce de câncer persistiu após controle para idade e variáveis socioeconômicas (BAIRROS et al., 2011). Observa-se que não há informação deste dado em $100 \%$ das coletas realizadas em Espinosa/MG. Este dado foi subnotificado pelo profissional que realizou o exame.

O exame citopatológico deve ser realizado após o início da atividade sexual (IAS) com intervalos semestrais no primeiro ano e, se normais, manter seguimento anual. A faixa etária constitui um indicador de saúde muito importante, pois quanto menor a idade de início da mulher para a realização do exame, mais precoce será o IAS. Ainda assim, o Ministério da Saúde (MS) recomenda captar, obrigatoriamente, mulheres entre 25-64 anos (BRASIL, 2011a; MELO et al., 2012). O intervalo de idade que se mostrou prevalente entre as mulheres deste município foi de 40-49 anos (32,4\%). Vale ressaltar que uma parcela mínima de 3,7\% realizou o exame com idade entre 15-19 anos e, consequentemente, iniciou a atividade sexual.

O método de rastreamento do CCU e de suas lesões precursoras é o exame citopatológico. $O$ intervalo entre os exames deve ser de três anos, após dois exames negativos, com intervalo anual (BRASIL, 2013). Neste estudo, as mulheres respeitam o intervalo preconizado de modo a realizar o exame anualmente. 
A técnica adequada da coleta da amostra se faz por meio da captação de células da ectocérvice com a espátula de Ayres e esfregando em metade da lâmina em sentido unidirecional (de cima para baixo) e células da endocérvice com a escovinha endovaginal de modo a esfregar na outra metade da lâmina em sentido rotativo unidirecional (da esquerda para a direita) (SILVA et al., 2014). Sendo assim, a técnica correta foi estabelecida pelos profissionais de saúde nestas mulheres de Espinosa com 100\% de sua adequabilidade satisfatória.

Achados laboratoriais com presença microbiológica de Lactobacillus sp e cocos constituem achados normais, pois fazem parte da microbiota e, na ausência de sinais e sintomas, sua presença não caracteriza infecção que necessite de tratamento (BRASIL, 2011b). Os resultados laboratoriais encontrados nas mulheres de Espinosa corroboram com o preconizado pelos estudos dos manuais do MS cuja prevalência microbiológica achada foi de Lactobacillus sp e de cocos.

O CCU é raro na adolescência, apresentando taxas de incidência de 0/100.000 adolescentes com idade entre 10 a 19 anos e de 1,7/100.000 mulheres com idade entre 20 e 24 anos. Entretanto, vários estudos mostram a incidência crescente das lesões pré-neoplásicas em idade cada vez menores. Em um relato de caso, foi observada a presença de carcinoma microinvasor do colo uterino em paciente de 14 anos, com menarca aos 10 anos, e a primeira relação aos 12 anos (GONÇALVES et al., 2009). Nos achados deste estudo ao qual diverge com o estudo anterior, houve a descoberta de duas pacientes cujos resultados constataram a presença de lesão intra-epitelial de alto grau (LIE-AG) e carcinoma epidermóide invasivo (CEI), ambas com idade entre 45-59 anos. Outros estudos corroboram estes achados (DISCACCIATI; BARBOZA; ZEFERINO, 2014).

O estudo demonstra também a ausência de resultados com confirmação de câncer do colo do útero (adenocarcinoma in situ [AIS] e adenocarcinoma invasor [AI]) inferindo, assim, na preocupação com a sua saúde e melhoria da qualidade de vida $(\mathrm{QV})$. 


\section{CONCLUSÃO}

A coleta do exame realizado no município de Espinosa atende aos requisitos dispostos pelo MS. A realização rotineira periódica do exame citopatológico contribui para o aumento da QV das mulheres. O entendimento da importância da realização deste exame pelas mulheres pode ser atribuído à captação das faltantes por meio da busca ativa feita pelo profissional por meio de um trabalho promocional. o tempo da realização da coleta, quanto da chegada do resultado, apresenta-se sem atrasos por parte do laboratório, conforme preconizado pelos manuais do MS, denotando uma análise laboratorial em tempo hábil com relação a sua realização. A instauração de um diagnóstico precoce é fundamental para o início de um possível tratamento.

Neste município não houveram casos diagnosticados de neoplasias intra-epitelial cervical (NIC) em estágios II e III. Dessa forma, infere-se sobre a preocupação e cuidados tidos pelas mulheres quanto a sua saúde. As coletas de exame preventivo atendem satisfatoriamente ao preconizado pela legislação vigente e ao perfil de prevenção do câncer de colo uterino nas mulheres, ainda que ocorram falhas no preenchimento dos dados da mulher na ficha de coleta por parte do profissional de saúde habilitado.

Salienta-se a importância do preenchimento de todos os dados da mulher na ficha de coleta a fim de poder traçar um melhor perfil epidemiológico, de modo a intervir em estratégias de promoção da saúde e prevenção da doença, bem como na busca pelas faltantes.

Este estudo, apesar de mostrar uma visão panorâmica estatística da realização do exame citopatológico pelas mulheres, a mesma apresenta uma restrição dos dados com relação ao período, ao local de realização e a disponibilização de registros na íntegra, fazendo com que os dados se limitem a uma análise local e puramente descritiva. É esperado a viabilização de outros estudos de caráter comparativo apresentando a discussão de outras variáveis não contempladas neste estudo por falta de registro durante o preenchimento da ficha de coleta.

\section{REFERÊNCIAS}

BAIRROS, F. S.; MENEGHEL, S. N.; DIAS-DA-COSTA, J. S.; BASSANI, D. G.; MENEZES, A. M. B.; GIGANTE, D. P.; OLINTO, M. T. A. Racial inequalities in access to women's health care in southern Brazil. Cadernos de Saúde Pública. Rio de Janeiro, v. 27, n. 12, p. 2364-2372, 2011. Disponível em: http://www.scielo.br/pdf/csp/v27n12/08.pdf 
BRASIL. Ministério da Saúde. Portal Brasil. Ministério da Saúde amplia faixa etária para rastreamento do câncer de colo de útero. Brasília: MS, 2011a. Disponível em: http://www.brasil.gov.br/saude/2011/07/ministerio-da-saudeamplia-faixa-etaria-para-rastreamento-do-cancer-de-colo-de-utero

BRASIL. Ministério da Saúde. Instituto Nacional do Câncer. Coordenação Geral de Ações Estratégicas. Divisão de Apoio à Rede de Atenção Oncológica. Diretrizes brasileiras para o rastreamento do câncer do colo do útero. Rio de Janeiro: $\quad$ INCA, 2011b. Disponível em: http://bvsms.saude.gov.br/bvs/publicacoes/inca/rastreamento_cancer_colo_utero.pdf

BRASIL. Ministério da Saúde. Secretaria de Atenção à Saúde. Departamento de Atenção Básica. Cadernos de atenção básica: controle dos canceres do colo do útero e da mama. Brasília: MS, 2013. Disponível em: http://bvsms.saude.gov.br/bvs/publicacoes/controle_canceres_colo_utero_2013.pdf

BRASIL. Ministério da Saúde. Instituto Nacional do Câncer. Papanicolau (exame preventivo de colo de útero). Rio de Janeiro: INCA, 2015a.

BRASIL. Ministério da Saúde. Departamento de Informática do Sistema Único de Saúde. Sistema de Informação da Atenção Básica. Cadastramento familiar - Brasil: mulheres de $\mathbf{1 5}$ a maiores de $\mathbf{6 0}$ anos de Espinosa, Minas Gerais. Brasília: DATASUS, 2015b.

DISCACCIATI, M. G.; BARBOZA, B. M. S.; ZEFERINO, L. C. Por que a prevalência de resultados citopatológicos do rastreamento do câncer do colo do útero pode variar significativamente entre duas regiões do Brasil? Revista Brasileira de Ginecologia e Obstetrícia. Rio de Janeiro, v. 36, n. 5, p. 192-197, 2014. Disponível em: http://www.scielo.br/pdf/rbgo/v36n5/0100-7203-rbgo-36-05-00192.pdf

FARIAS, A. C. B.; BARBIERI, A. R. Seguimento do câncer de colo de útero: estudo da continuidade da assistência à paciente em uma região de saúde. Escola Anna Nery Revista de Enfermagem. Rio de Janeiro, v. 20, n. 4, p. 1-9, 2016. Disponível em: http://www.scielo.br/pdf/ean/v20n4/1414-8145-ean-20-04-20160096.pdf

GONÇALVES, C. V.; QUINTANA, S. M.; MARCOlin, A. C.; DUARTE, G.; COSTA, J. S. D.; KARAM, F.; BIANCHI, M. S. Microinvasive carcinoma of the uterine cervix in a 14-year-old adolescent: case report and literature review. São Paulo Medical Journal. São Paulo, v. 127, n. 2, p. 105-107, 2009. Disponível em: http://www.scielo.br/pdf/spmj/v127n2/a10v1272.pdf

INSTITUTO BRASILEIRO DE GEOGRAFIA E ESTATÍSTICA. Infográficos: dados gerais do município de Espinosa, Minas Gerais. Rio de Janeiro: IBGE, 2016.

MELO, M. C. S. C.; VILELA, F.; SALIMENA, A. M. O.; SOUZA, I. E. O. O enfermeiro na prevenção do câncer do colo do útero: o cotidiano da atenção primária. Revista Brasileira de Cancerologia. Rio de Janeiro, v. 58, n. 3, p. 389-398, 2012. Disponível

em:

http://www1.inca.gov.br/rbc/n_58/v03/pdf/08_artigo_enfermeiro_prevencao_cancer_colo_utero_cotidiano_atenc ao_primaria.pdf

RIBEIRO, L.; BASTOS, R. R.; VIEIRA, M. T.; RIBEIRO, L. C.; TEIXEIRA, M. T. B.; LEITE, I. C. G. Rastreamento oportunístico versus perdas de oportunidade: não realização do exame de Papanicolaou entre mulheres que frequentaram o prénatal. Cadernos de Saúde Pública. Rio de Janeiro, v. 32, n. 6, p. 1-13, 2016. Disponível em: http://www.scielo.br/pdf/csp/v32n6/1678-4464-csp-32-06-e00001415.pdf

SILVA, D. S. M.; SILVA, A. M. N.; BRITO, L. M. O.; GOMES, S. R. L.; NASCIMENTO, M. D. S. B.; CHEIN, M. B. C. Rastreamento do câncer do colo do útero no Estado do Maranhão, Brasil. Ciência \& Saúde Coletiva. Rio de Janeiro, v. 19, n. 4, p. 1163-1170, 2014. Disponível em: http://www.scielo.br/pdf/csc/v19n4/1413-8123-csc-19-04-01163.pdf

\section{(cc) Br}

Este trabalho está licenciado com uma Licença Creative Commons - Atribuição 4.0 Internacional. 